\title{
Finite element analysis for normal pressure hydrocephalus: the effects of the integration of sulci
}

\author{
Hakseung Kim ${ }^{a}$, Dae-Hyeon Park ${ }^{\mathrm{a}}$, Seong Yi ${ }^{\mathrm{a}, \mathrm{b}}$, Eun-Jin Jeong ${ }^{\mathrm{a}}$, Byung C. Yoon ${ }^{\mathrm{c}}$, Marek Czosnyka ${ }^{\mathrm{d}}$, \\ Michael PF Sutcliffe, ${ }^{\mathrm{e}}$ and Dong-Joo Kim ${ }^{\mathrm{a}, \mathrm{d}, *}$ \\ ${ }^{\text {a}}$ Department of Brain and Cognitive Engineering, Korea University, Seoul, South Korea. \\ ${ }^{b}$ Department of Neurosurgery, Yonsei University College of Medicine, Seoul, South Korea. \\ ${ }^{c}$ Department of Neurosurgery, Stanford University School of Medicine, Stanford, California, USA. \\ dDepartment of Neurosurgery, Addenbrooke's Hospital, University of Cambridge, Cambridge, United Kingdom. \\ ${ }^{\mathrm{e}}$ Department of Engineering, University of Cambridge, Cambridge, United Kingdom.
}

*Address correspondence to:

Dong-Joo Kim, Ph.D.

Departments of Brain and Cognitive Engineering, Korea University,

Anam-dong, Seongbuk-gu, Seoul, South Korea 136-713

Phone: 0082-2-3290-5929

E-mail: dongjookim@korea.ac.kr 


\begin{abstract}
Finite element analysis (FEA) is increasingly used to investigate the brain under various pathological changes. Although FEA has been used to study hydrocephalus for decades, previous studies have primarily focused on ventriculomegaly. The present study aimed to investigate the pathologic changes regarding sulcal deformation in normal pressure hydrocephalus (NPH).

Two finite element (FE) models - an anatomical brain geometric (ABG) model and the conventional simplified brain geometric (SBG) model—of NPH were constructed. The models were constructed with identical boundary conditions but with different geometries. The ABG model contained details of the sulci geometry, whereas these details were omitted from the SBG model. The resulting pathologic changes were assessed via four biomechanical parameters: pore pressure, von Mises stress, pressure, and void ratio. NPH was induced by increasing the transmantle pressure gradient (TPG) from 0 to a maximum of $2.0 \mathrm{mmHg}$.

Both models successfully simulated the major features of NPH (i.e., ventriculomegaly and periventricular lucency). The changes in the biomechanical parameters with increasing TPG were similar between the models. However, the SBG model underestimated the degree of stress across the cerebral mantle by $150 \%$ compared with the ABG model. The SBG model also overestimates the degree of ventriculomegaly (increases of $194.5 \%$ and $154.1 \%$ at $\mathrm{TPG}=2.0 \mathrm{mmHg}$ for the $\mathrm{SBG}$ and $\mathrm{ABG}$ models, respectively).

Including the sulci geometry in a FEA for NPH clearly affects the overall results. The conventional SBG model is inferior to the ABG model, which accurately simulated sulcal deformation and the consequent effects on cortical or subcortical structures. The inclusion of sulci in future FEA for the brain is strongly advised, especially for models used to investigate space-occupying lesions.
\end{abstract}

Key words: biomechanics; bi-phase; finite element model; transmantle pressure gradient; normal pressure hydrocephalus. 


\section{Introduction}

Hydrocephalus is a collective term for the excessive accumulation of cerebrospinal fluid (CSF) in cerebral ventricles, which can have many causes. Hydrocephalus commonly accompanies increased intracranial pressure (ICP) and is associated with well-known symptoms, including headache, nausea, vomiting and cognitive deterioration. However, in normal pressure hydrocephalus (NPH), there is an accumulation of CSF without an increase in ICP. Common symptoms of NPH include the classic 'triad' of gait disturbance, urinary incontinence and cognitive deterioration (Adams et al., 1965; Pople, 2002). Despite the severe symptoms, treatment of NPH is limited. One treatment option is the surgical diversion of the accumulated CSF; however, this procedure does not 'cure' the disease. For wide variety of reasons (e.g., mechanical failure, infection, occlusion, under- or overdrainage), shunt insertion results in a high failure rate, ranging from $30 \%$ from $50 \%$, and NPH patients suffer from an excessively higher failure rate (Dusick et al., 2008; Faggin et al., 2011; Feng et al., 2004; Malm et al., 2004; Stein and Guo, 2008). Further efforts should be made for better understanding NPH.

Finite element analysis (FEA) offers high flexibility for studying the biomechanical behavior of NPH in the brain, albeit within the constraints inherent to clinical studies. Substantial efforts have been made to study hydrocephalus using finite element (FE) models. The first study to apply FEA to describe hydrocephalus was published in 1987 (Nagashima et al., 1987); since then, the FE models have been continually improved with the technological advancements in neuroimaging and computation (Lefever et al., 2013). Nonetheless, the majority of previously reported FE models primarily focused on the most prominent feature of hydrocephalus, i.e., ventriculomegaly (Cheng and Bilston, 2010; Clatz et al., 2007; Dutta-Roy et al., 2008; Lefever et al., 2013; Wilkie et al., 2010, 2012; Wirth and Sobey, 2006). However, the deformation of brain tissue caused by NPH not only induces the dilation of ventricles but also often extends to the sulcal effacement.

The omission of sulcal deformation in previous hydrocephalus FE models may have been intended to reduce the computational complexity. However, recent FEA studies for traumatic brain injury suggest that the structural characteristics of the sulci affect several properties of the brain under mechanical stress (Cloots et al., 2008; Ho and Kleiven, 2009). The overall cerebral dysfunction associated with NPH may be partially induced by sulcal effacement. The presented study aimed to investigate the degree of tissue distortion that is caused by the mass effects of different temporal and spatial pathogenic profiles in a hydrocephalic model with and without sulci. 


\section{Materials and methods}

\subsection{Finite element model}

Simulation was performed under two-dimensional plane strain conditions using Abaqus (Abaqus/Standard, Dassault Systèmes, France). Although a full three-dimensional model with the sulcal geometric details would provide more realistic results compared to a two-dimensional model (Cheng and Bilston, 2010), it would also involve immense computational complexity. The present study is a preliminary analysis on a specific subject (i.e., the effects of the sulcal geometric details in an FE model for NPH); thus, the use of a two-dimensional model was considered appropriate. A schematic model is provided in Fig. 1. A series of $\mathrm{T}_{2}$-weighted magnetic resonance (MR) images of a healthy male subject were collected (SIEMENS MAGNETOM TRIO) at the Korea University Brain Imaging Center. The use of the MR image was approved by an institutional review board (1040548-KU-IRB-14-83-A-1).
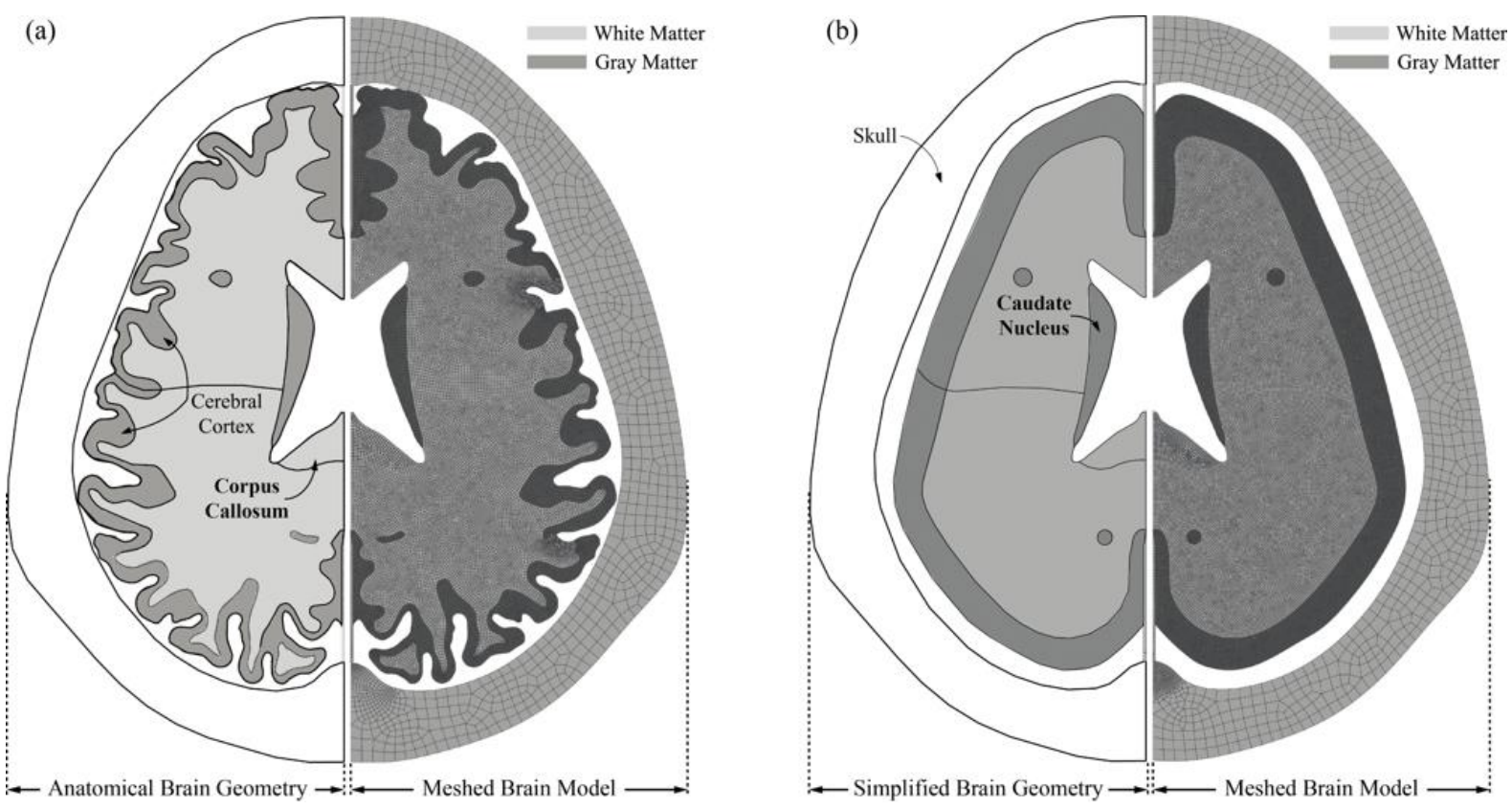

Fig. 1. An overview of the anatomical brain geometry and the simplified brain geometry.

To acquire the geometry, the regions of interest (ROI) from the brain image were segmented using ScanIP (Simpleware Software Ltd., U.K.). The brain image slices obtained were then meshed using ScanFe with threedimensional finite elements. Because the MR images are composed of voxels, the images cannot be directly used for two-dimensional analysis. Therefore, a customized FORTRAN code was written to derive a twodimensional surface from the voxels with a finite thickness. The code retrieved the surface node and element 
information from the meshed three-dimensional slice, which allowed the construction of a two-dimensional model based on the obtained information. The model mesh obtained was further refined using Hypermesh (Altair Engineering, USA). Finally, the pre-processed model was imported to ABAQUS for analysis. The resulting mesh is a two-dimensional mesh without thickness.

Various anatomical features were included within the models in two strands. The physical regions included the gray/white matter, the falx cerebri and the skull. The interface regions included the outer layer of the cerebral cortex, the ependyma and the dura at the contact. From the geometry model, the center line was taken as the plane of symmetry. Therefore, only half of each slice was needed for the analysis. In addition, the conventional simplified brain geometric (SBG) model was constructed by interpolating several 'peaks' along the sulci and adjusting the area according to that of the newly proposed anatomical brain geometric (ABG) model. This model was used to minimize the difference in gross areas between the peripheral CSF space $(0.1 \%)$ and the cortex $(0.7 \%)$ of ABG and SBG. The gross areas of lateral ventricle, corpus callosum, caudate nucleus and white matter were identical. Though the geometrical morphologies of the two models are different, the difference in the gross areas of anatomical structures in the two models was negligible. Consequentially, the comparison of these models is valid.

Four-node quadrilateral pore fluid flow elements, including CPE4RP (for pore pressure plane strain elements) and CPE4R (for plane strain elements), were used with reduced-integration; otherwise, three-node triangular solid elements for plane strain elements (“CPE3”) were used. Implicit time integration associated with the poro-elastic and hyper-elastic material analyses was performed for the non-linear geometric analysis using Abaqus 6.14.

To implement the model in Abaqus, the brain tissue is regarded as a crushable porous foam with an initial void ratio of 0.25 (Nagashima et al., 1987), enabling a simulation of fluid behavior. Ideally, the brain tissue matrix should have no initial pressure in the model, as the brain is filled with fluid (e.g., interstitial fluid). The standard poro-elastic consolidation theory was considered suitable for the fluid phase of the model (Biot, 1941), whereas the solid phase was considered a compressible, neo-Hookean hyper-elastic material and modeled accordingly (Dutta-Roy et al., 2008). The inhomogeneity of the brain tissue within the parenchyma (i.e., differences between the gray and white matter) was also considered. Additional details regarding the material properties are summarized in Table 1. 


\section{Table 1}

Details of the material properties utilized for the model

\begin{tabular}{|c|c|c|c|c|c|c|c|}
\hline \multicolumn{4}{|c|}{ Parenchyma } & \multicolumn{4}{|c|}{ Falx cerebri } \\
\hline $\bar{~} \overline{E_{p}[\mathrm{~Pa}]}$ & 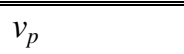 & $\overline{C_{10}[\mathrm{~Pa}]}$ & $\overline{D_{I}[P a]}$ & $\overline{E E_{f}[P a]}$ & $\overline{v_{f}}$ & $\begin{array}{ll}\rho_{f} & {\left[\mathrm{kgm}^{-3}\right]}\end{array}$ & $\bar{t} t[\mathrm{~mm}]$ \\
\hline 420.5 & 0.35 & 77.9 & $4.28 \times 10^{-3}$ & $3.4 \times 10^{6}$ & 0.45 & 1130 & 2 \\
\hline$e$ & $\rho_{p}\left[k g m^{-3}\right]$ & \multicolumn{2}{|l|}{$k[\mathrm{~m} / \mathrm{s}]$} & \multicolumn{4}{|l|}{ Skull } \\
\hline \multirow[t]{2}{*}{0.25} & \multirow[t]{2}{*}{1040} & WM & $1 \times 10^{-7}$ & $E_{s}[P a]$ & $v_{s}$ & $\rho_{s}\left[\mathrm{kgm}^{-3}\right]$ & \\
\hline & & GM & $1 \times 10^{-9}$ & $14 \times 10^{9}$ & 0.23 & 1412 & \\
\hline
\end{tabular}

$C_{10}$ and $D_{l}$ were calculated from $E$ and $v$. For the complete derivation of parameters, refer to the APPENDIX.

The skull and the falx cerebri were considered linear-elastic materials. The Poisson's ratio of the 'drained' porous brain solid material is considered to be 0.35 . However, since the brain model is always saturated with incompressible fluid (Poisson's ratio $=0.5$ ), the effective Poisson's ratio of the overall brain model combining solid and fluid elements becomes nearly 0.5 , which makes it nearly incompressible material. $E_{p}=$ Young's modulus of the parenchyma (Dutta-Roy et al., 2008; Miller et al., 2005); $v_{p}=$ Poisson's ratio of the parenchyma (Kaczmarek et al., 1997a; Nagashima et al., 1987; Pena et al., 1999); $k=$ permeability (Kaczmarek et al., 1997b); $e=$ void ratio (Nagashima et al., 1987); $\rho_{p}=$ density of the parenchyma; $E_{f}=$ Young's modulus of the falx cerebri (Jiroušek et al., 2005; Takhounts, 2003); $v_{f}=$ Poisson's ratio of the falx cerebri (Jiroušek et al., 2005; Yoganandan, 1998); $\mathrm{t}=$ the thickness (Yoganandan, 1998); $\rho_{f}=$ density of the falx cerebri (Yoganandan, 1998); $E_{s}=$ Young's modulus of the skull (Jiroušek et al., 2005; Takhounts, 2003); $v_{f}=$ Poisson's ratio of the skull (Jiroušek et al., 2005; Yoganandan, 1998); $\rho_{s}=$ density of the skull (Yoganandan, 1998).

To assess the accuracy and convergence of the solutions from each model, the strain energy of the elements was computed. The SBG model, which was initially comprised of 842 coarse elements, was refined by halving its element size in each step, until its element number reached 41,202. The ABG model, which was initially comprised of 818 coarse elements, underwent the same process until the element number reached 43,754 (Fig. 2). The strain energy converged to the approximately 40,000 element models. It was concluded that further mesh refinement would not produce better answers, therefore, 41,202 and 43,754 element models were selected for the SBG and ABG models, respectively. As it was evident that excessive mesh refinement of the cranium was not necessary, further adjustments were made to decrease computational complexity. After the increase in element size for the cranium in both models, the final numbers of elements used became 30,260 in the ABG model and 30,512 in the SBG model (Fig. 1). The effect of reducing of the element number in the cranium was negligible in both models. 

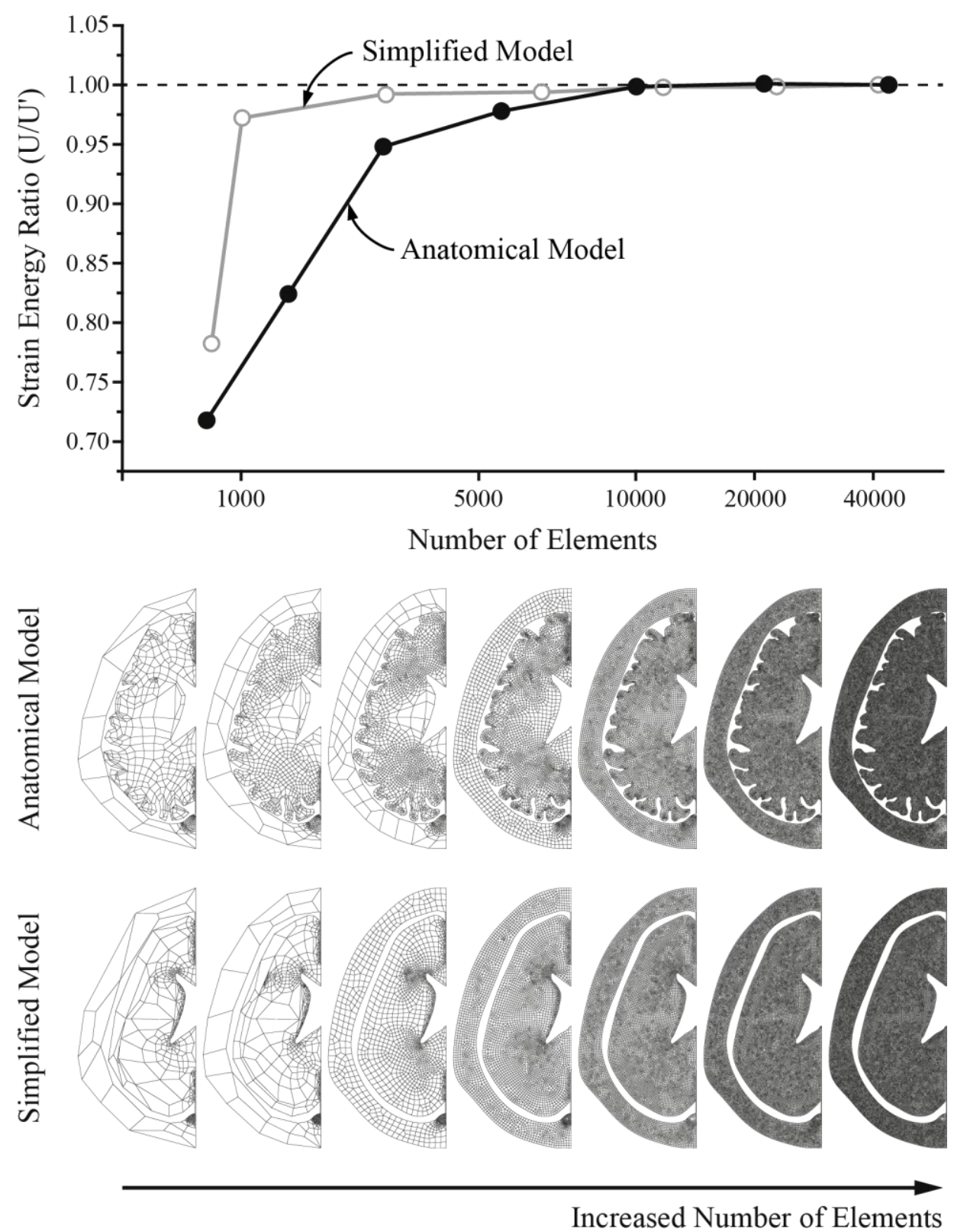

Fig. 2. Log plots depicting the convergence of strain energy as a function of the number of elements used in the two models (upper panel). U indicates the strain energy of each model at various numbers of element points, and $U$ ' indicates the strain energy at each of the most refined models. The difference in strain energy becomes negligible after the element number reaches approximately 10,000 for both models. The actual structures of both models are presented with increasing element number (lower panel).

\subsection{Brain loading and boundary conditions}

The causes of ventriculomegaly can vary from aqueduct stenosis to idiopathic ventricular fluid accumulation found in the NPH. In the present study, we assumed a recently proposed theory, which suggests that the primary cause of ventriculomegaly in NPH is the gradual change in transmantle pressure (Greitz, 2004) rather than an increase in ventricular fluid pressure. 
To implement the theoretical conditions in the model, the drainage of interstitial fluid through capillaries was modeled (Greitz, 1993; Greitz et al., 1997). The outer boundary of the cerebral mantle was assumed to be the capillary region. To shrink cerebral mantle owing to ventriculomegaly, interstitial fluid (ISF) should be drained out of the mantle, considering the incompressible characteristics of water. ISF flows freely across the capillary; thus, the pore pressure was set to zero.

The initiation of ventricular enlargement was induced by adjusting the transmantle pressure (TPG; defined as the difference between the capillary pressure and the ventricular fluid pressure). In this study, the relationship between the increase in the initial level of TPG and the degree of the subsequent deformation was also analyzed by subjecting the two models (i.e., the SBG model and the ABG model) to varying levels of TPG $(0.1,1.0$ and $2.0 \mathrm{mmHg}$ ). The level of TPG was considered sufficient to initiate NPH (Dutta-Roy et al., 2008; Hoff and Barber, 1974), unlike in non-communicating hydrocephalus in which immensely increased ventricular fluid pressure causes significantly high TPG. In each case, the TPG increased over 340,000 seconds, the equivalent to four days (Nagashima et al., 1987). The radiologic features closely associated with NPH are ventriculomegaly, periventricular lucency, and sulcal effacement (Chan et al., 2003). The four key biomechanical parameters closely associated with these features are pore pressure, von Mises stress, pressure, and void ratio. A void ratio over 0.25 was interpreted as tissue expansion due to the fluid accumulation; a pore pressure of zero or a negative pore pressure was interpreted as free fluid flow or fluid accumulation.

A coupled pore fluid flow and stress analysis algorithm was used. Non-linear and asymmetric solver options were used due to the presence of the crushable but fully saturated foam model for the cerebral mantle. The major factors related to the model convergence were the contact instability and element distortion.

\section{Results}

The FEA for both models produced the key features of NPH, including ventriculomegaly and periventricular lucency. The most prominent difference between the two models was the area in which the CSF accumulated (Fig. 3). 

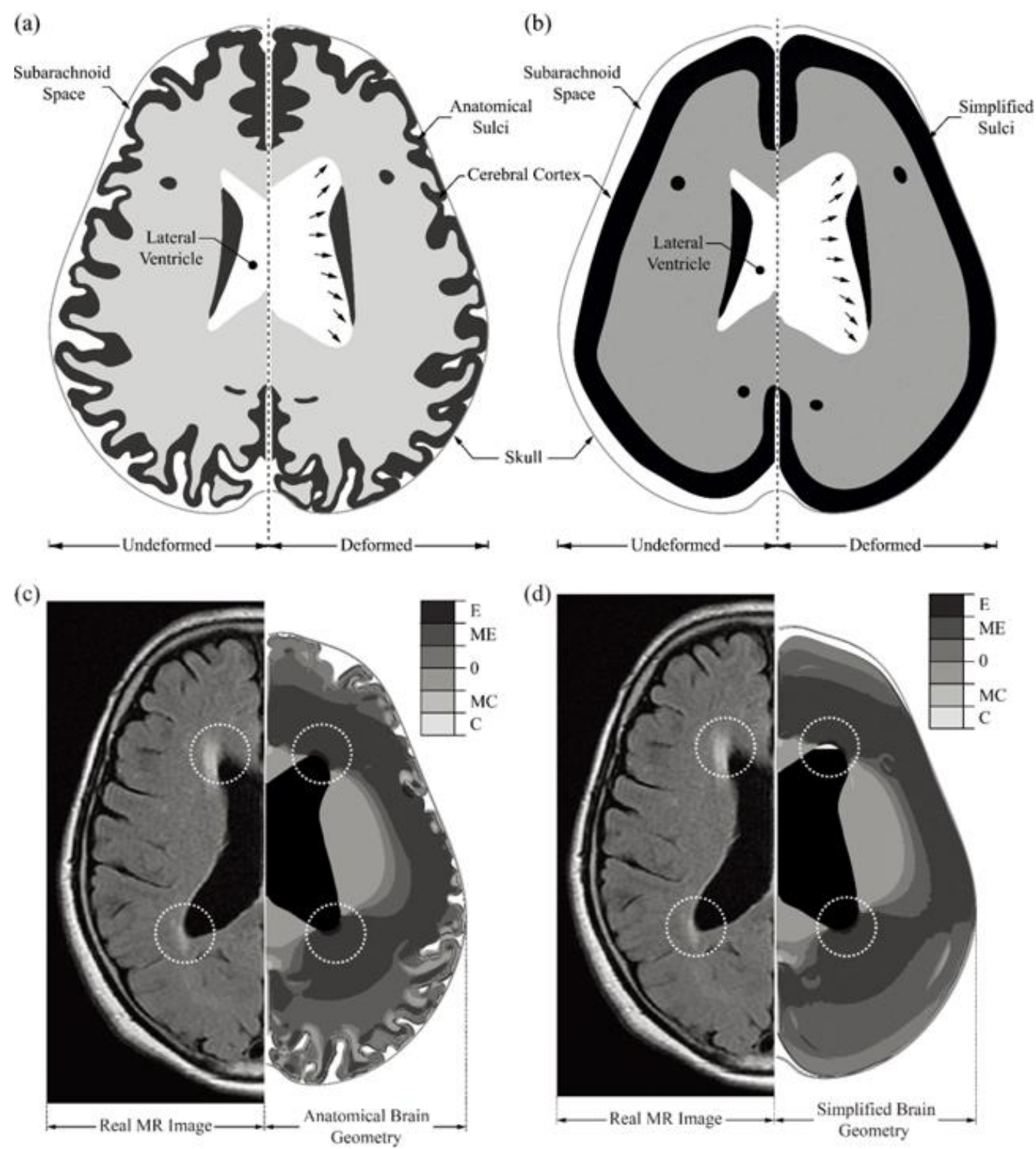

Fig. 3. Comparison of the ABG and SBG models (Upper panel A). The ABG model before and after the increase of TPG (Upper panel B). The SBG model before and after the increase of TPG (Lower panels C, D). Validation of the two models with an actual MR image of an NPH patient depicted in contour plots of volumetric strain. The increased volumetric strain implies that the expansion of the extracellular spaces due to the accumulation of interstitial fluid results in positive volumetric strain, and vice versa. $\mathbf{A B G}=$ anatomical brain geometry $\mathbf{C}=$ compression $\mathbf{E}=$ expansion; $\mathbf{M C}=$ mild compression $; \mathbf{M E}=$ mild expansion; $\mathbf{S B G}=$ simplified brain geometry; $\mathbf{T P G}=$ transmantle pressure gradient.

Fig. 3 depicts the initial and deformed shapes for the two models with a TPG of $2.0 \mathrm{mmHg}$. The structural deformation due to the increased TPG was most severe in the lateral ventricle, as both models simulated a ventricular expansion greater than $150 \%$ (194.5\% in the SBG model and $154.1 \%$ in the ABG model). In contrast, the subarachnoid space exhibited a large reduction (estimated as $89.3 \%$ in the SBG model and $71.5 \%$ 
in the ABG model). As depicted in Fig. 3 and Table 2, the ventricle was more dilated in the SBG model than in the ABG model, whereas the degree of areal reduction in the subarachnoid space (SAS) was more significant in the SBG model. The models also showed minor structural deformations across the intracranial area (Table 2).

\section{Table 2}

Structural deformations predicted by the ABG and SBG models

\begin{tabular}{|l|l|l|l|l|l|l|l|}
\hline \hline & SAS & LV & Cortex & CC & CN & WM & GM \\
\hline \hline SBG & $-89.3 \%$ & $194.5 \%$ & $0.2 \%$ & $-8.2 \%$ & $-3.1 \%$ & $-0.1 \%$ & $-0.1 \%$ \\
\hline ABG & $-71.5 \%$ & $154.1 \%$ & $-0.1 \%$ & $-4.2 \%$ & $-2.7 \%$ & $-0.1 \%$ & $-0.3 \%$ \\
\hline
\end{tabular}

Values are given as the percentage difference between the initial area and the area at TPG $=2.0 \mathrm{mmHg}$. SAS $=$ subarachnoid space; $\mathbf{L V}=$ lateral ventricle; $\mathbf{C C}=$ corpus callosum; $\mathbf{C N}=$ caudate nucleus; $\mathbf{W M}=$ white matter; $\mathbf{G M}=$ gray matter.

\subsection{Differences between the ABG and the SBG biomechanical parameters: ependymal wall}

The steady increase in the TPG from 0 to $2.0 \mathrm{mmHg}$ induced an increase in biomechanical parameters at the ependymal wall to varying degrees (Fig. 4).
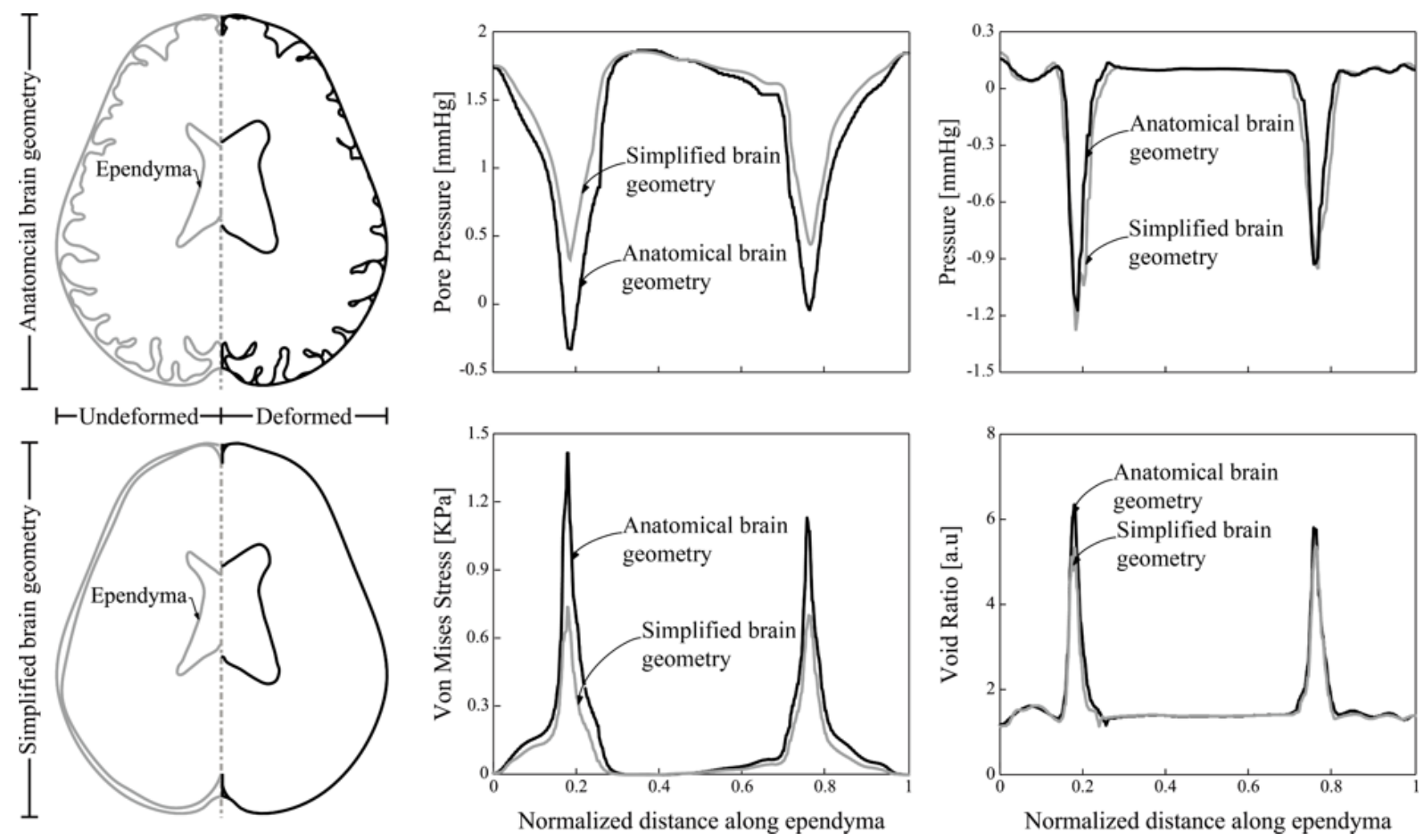

Fig. 4. The behaviors of biomechanical parameters at the ventricular ependymal wall in the SBG and ABG models with TPG at $2.0 \mathrm{mmHg}$. The two peaks in the four line graphs are the products of the geometrical features of the lateral ventricle (i.e., the anterior and posterior horns). 
The initial value of pore pressure at the ependymal wall was close to zero in both models. At TPG $=2.0$ $\mathrm{mmHg}$, the pore pressure in the SBG model was $0.9 \mathrm{mmHg}$, whereas the ABG model simulated a pore pressure of $1.2 \mathrm{mmHg}$ (Table 3). Although other biomechanical parameters (e.g., von Mises stress, pressure and void ratio) also increased with an increasing TPG, the degree of increase in values was significantly higher in the SBG model than in the ABG model. With TPG increased from 0.1 to $2.0 \mathrm{mmHg}$, the von Mises stress at the ependymal wall exponentially increased in the SBG and ABG models. However, the void ratio remained relatively constant, resulting in only $13.5 \%$ and $4.6 \%$ increases for the SBG and the ABG models, respectively. The findings of the ependymal wall analyses are summarized in Table 3.

Table 3

Changes in the biological parameters at the ependymal wall

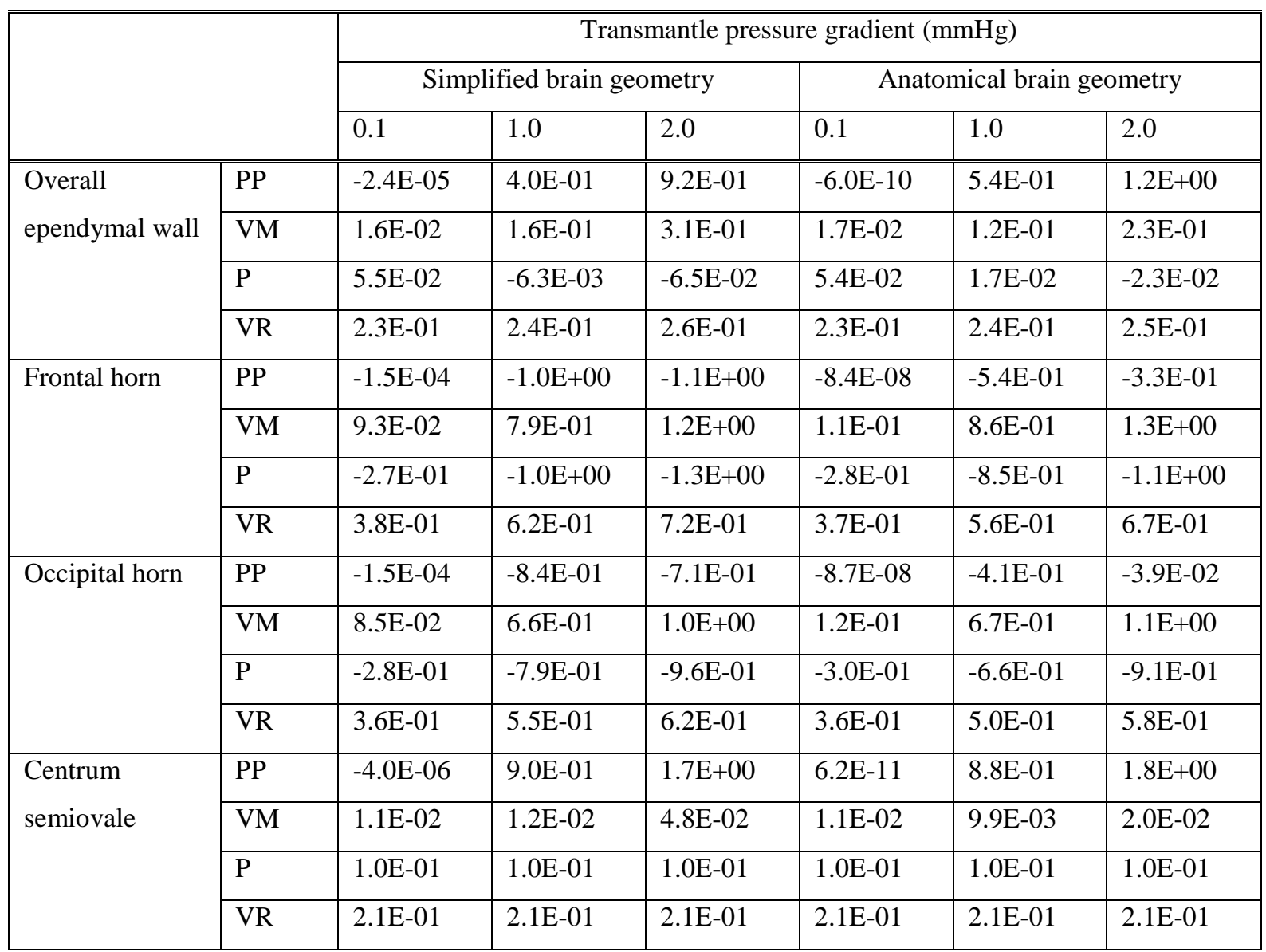

$\mathbf{P P}=$ pore pressure $(\mathrm{mmHg}) ; \mathbf{V M}=$ von Mises stress $(\mathrm{kPa}) ; \mathbf{P}=$ pressure $(\mathrm{mmHg}) ; \mathbf{V R}=$ void ratio $($ a.u $)$. 
The increase in the TPG had a varying effect on the biomechanical parameters. Although small discrepancies existed, the alterations in the pressure and void ratio occurred before the TPG reached $2.0 \mathrm{mmHg}$, whereas the increase in von Mises stress and pore pressure continued as the TPG was increased to $2.0 \mathrm{mmHg}$ (Fig. 5).

(a)
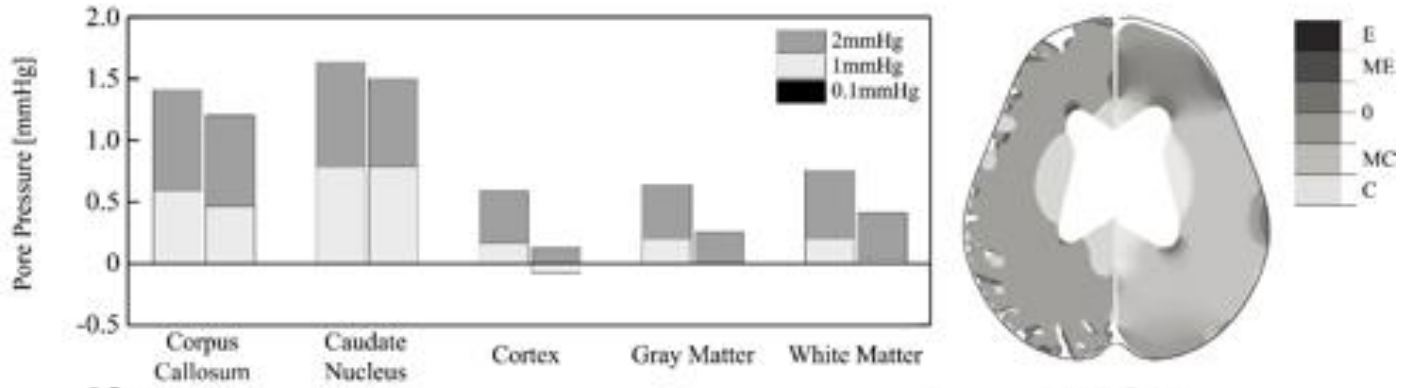

(b)
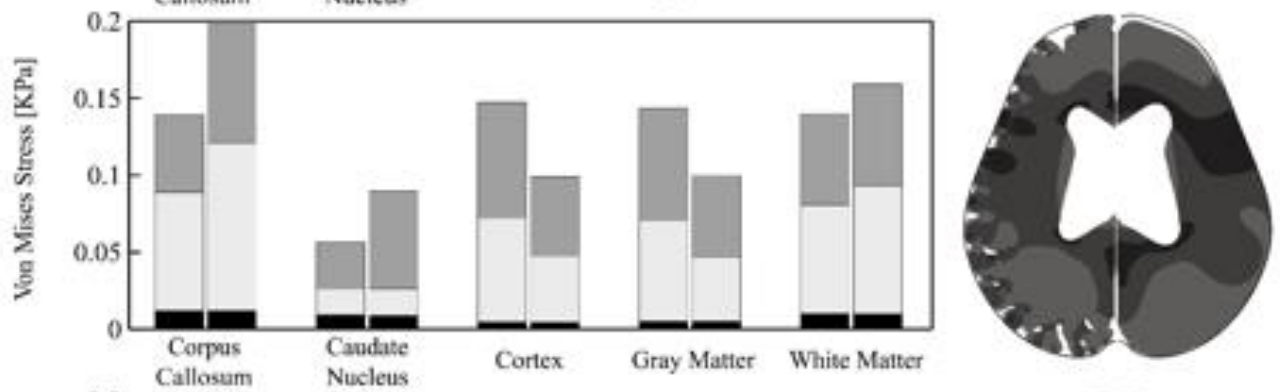

(c)
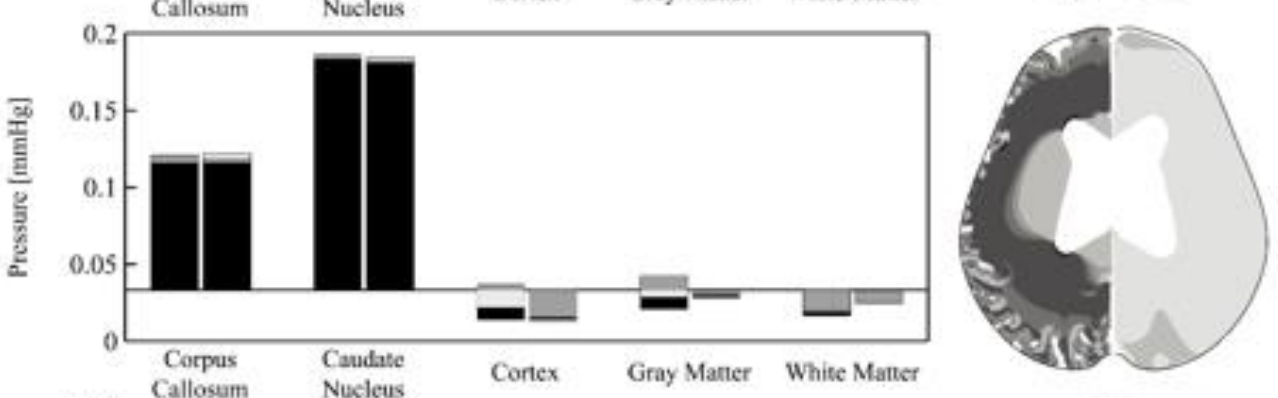

(d)
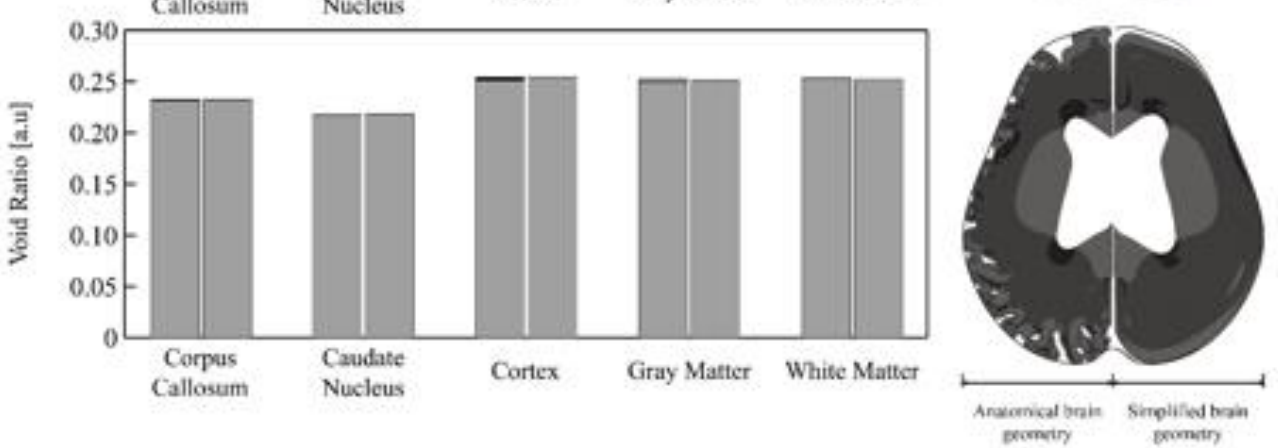

Fig. 5. Bar graphs showing the changes in the biomechanical parameters induced by the increase in the TPG for the ABG model (left bars) and the SBG model (right bars). Black = values calculated at TPG $=0.1 \mathrm{mmHg}$; Gray $=$ values calculated at $\mathrm{TPG}=1.0 \mathrm{mmHg}$; Dark gray $=$ values calculated at $\mathrm{TPG}=2.0 \mathrm{mmHg} . \mathrm{E}, \mathrm{ME}, \mathrm{MC}$ and $\mathrm{C}$ in the contour plots stand for expansion, mild expansion, mild compression, and compression, respectively. All values given are mean values. 
In both models, the pore pressure and von Mises stress increased as the TPG increased. The pore pressure, however, remained essentially unchanged at the initial phase of TPG increase (from 0 to $0.1 \mathrm{mmHg}$ ). As the TPG reached 1.0 and then $2.0 \mathrm{mmHg}$, the pore pressure across the intracranial space increased (Fig. 5). In general, the SBG model slightly underestimated the degree of increase for the pore pressure.

The degree of increase in von Mises stress was significantly lower in the SBG model compared to in the ABG model (Fig. 5). Additionally, in the ABG model, a small increase in the TPG (from 0.1 to $1.0 \mathrm{mmHg}$ ) was enough to cause a significant increase in the tissue stress level across the entire intracranial space, especially in structures near the sulci. Similar changes were found in the SBG model; however, the magnitude of the initial increase in the SBG model was significantly overestimated compared to the magnitude in the ABG model (Fig. $6)$.

The void ratio and pressure decreased as the TPG increased. A void ratio of less than 0.25 was observed in structures near the lateral ventricle (i.e., the corpus callosum and the caudate nucleus) in both models. However, the cortex retained a void ratio of approximately 0.25 regardless of the increase in the TPG. The near-ventricle structures exhibited a negative pressure immediately after the initial increase in the TPG (from 0 to $0.1 \mathrm{mmHg}$ ). Although small changes continued to occur as the TPG increased to $2.0 \mathrm{mmHg}$, the effects were minimal. In contrast, the initial increase in the TPG induced a positive pressure, which remained positive until the TPG increased to $1.0 \mathrm{mmHg}$. 
(a)

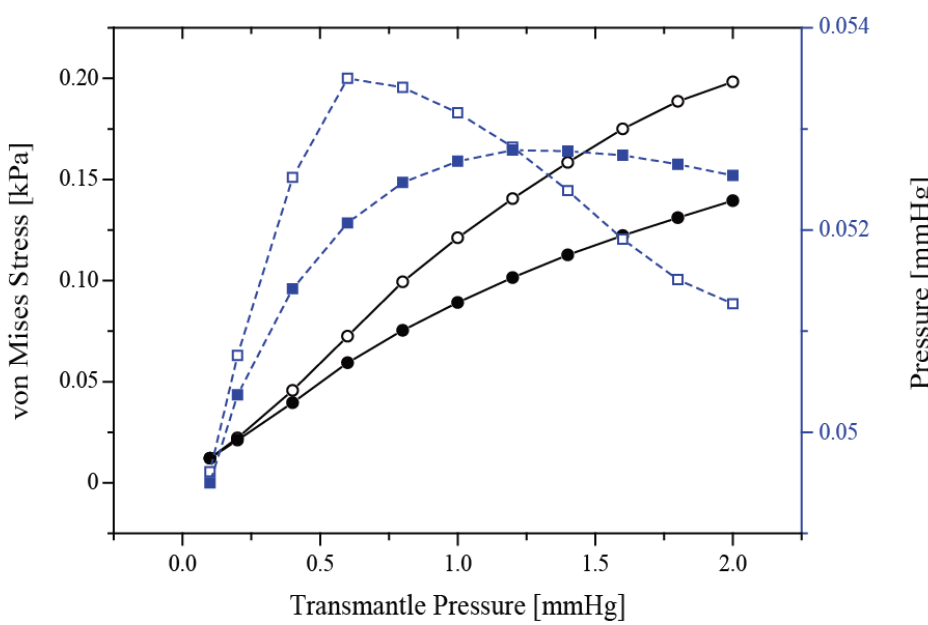

(b)

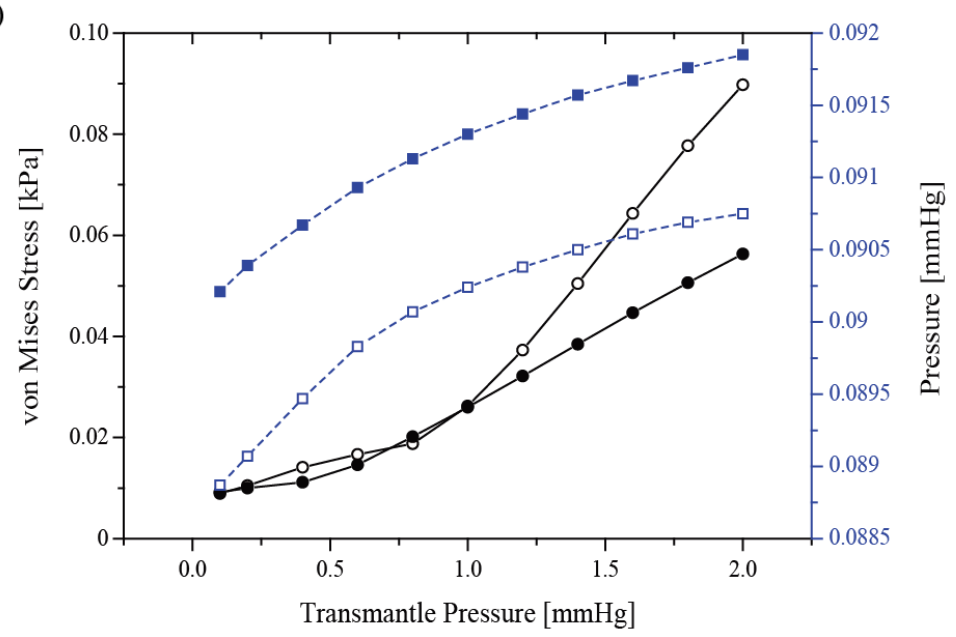

(c)

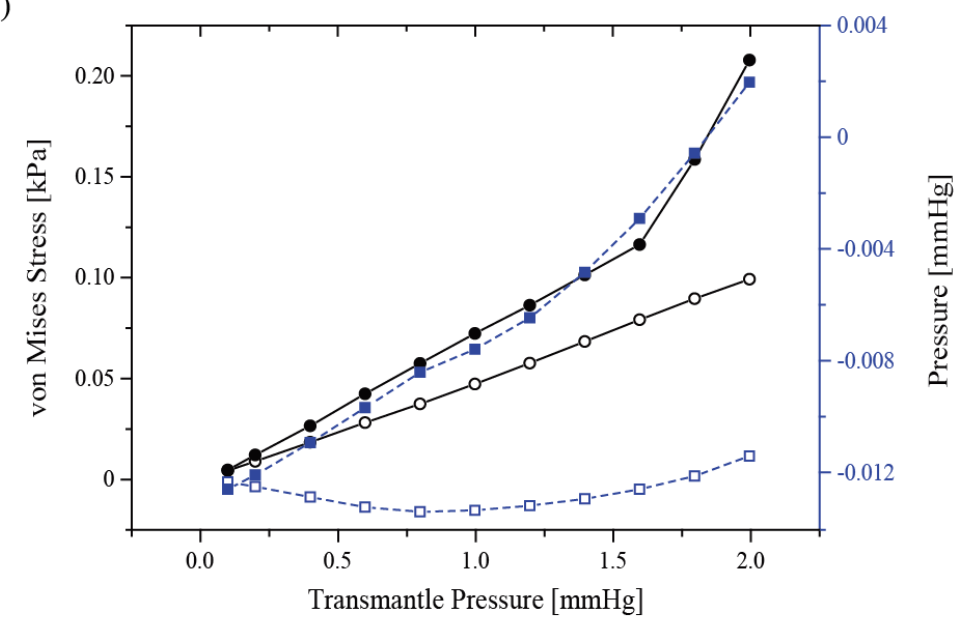

Von Mises Stress

calculated by simplified brain geometry

calculated by anatomical brain geometry

Pressure

Pressure

calculated by anatomical brain geometry $\quad \square$ calculated by simplified brain geometry

Fig. 6. Trends in the pressure and stress at (a) the corpus callosum, (b) caudate nucleus and (c) cerebral cortex according to the increasing TPG. $\mathrm{ABG}=$ anatomic brain geometry; SBG = simplified brain geometry 


\section{Discussion}

The inclusion of sulci in a brain FE model influences the biomechanical response during inertial acceleration simulations. In the present study, the ABG and SBG models were compared to investigate the importance of accurate geometrical modeling, including the sulci, in NPH. The loads and boundary conditions were equivalent for the models with and without sulci. However, the mechanism of ventricular dilation remains a controversial subject, and therefore, the boundary conditions used in the models require further explanation.

Although current FEA studies generally adopt the classical theory regarding CSF dynamics (i.e., ventricular dilation solely due to an obstruction in the CSF pathway or excessive secretion), this theory has been recently challenged (Battal et al., 2011; Bulat et al., 2008; Greitz and Hannerz, 1996; Stephensen et al., 2002). Because the brain parenchyma is nearly incompressible, ventriculomegaly can occur only if there is enough space in the intracranial system. A reduction in the volume of brain parenchyma, rather than increased ventricular fluid pressure alone, is needed for ventriculomegaly to develop (Greitz, 1993; Greitz, 2004), and a TPG is required for this reduced volume to occur. Once the initial increase of TPG causes pathologic changes, the pressure will be equally distributed throughout the intracranial space. Therefore, it is possible that ventriculomegaly persists without a TPG.

\subsection{Sulcal deformation in the $A B G$ and the $S B G$ models}

With the incorporation of TPG changes, the analyses successfully simulated brains in a hydrocephalic state (Fig. 3). The ABG and SBG models both showed enlarged ventricles and compressed SAS, which are common radiological findings in NPH patients (Kitagaki et al., 1998). The areal decrease in SAS was more severe in the SBG model than in the ABG model (Table 2), most likely due to sulcal deformation. As illustrated in Fig. 3, the increased TPG resulted in a moderate obliteration of the sulci in the ABG model. This result deserves attention because the obliterated sulci are positively associated with a good outcome after surgical CSF diversion treatment (Poca et al., 2004).

The sulcal obliteration of the ABG was derived by manipulating only one physiological condition, i.e., the TPG. Although the pathogenesis of NPH is largely unknown, it may be possible that the symptoms of NPH patients presenting with obliterated sulci are primarily due to an increase in the TPG. If this were the case, the surgical CSF diversion, resulting in a decrease in the TPG, would likely be beneficial. Nonetheless, the obliteration of the sulci is not always present. In fact, dilated or normal sulci are often found in the neuroimages of NPH patients. In any case, the radiographic signs of sulcal deformation are considered important diagnostic 
clues in NPH (Adachi et al., 2006; Iseki et al., 2014); however, only a few studies exist regarding the relationship between sulcal deformation and the space-occupying lesion (Bradshaw et al., 2001; Mutoh et al., 1988). The relationship between shape variations and the degree of sulcal deformation and clinical outcomes requires further investigation.

\subsection{Ependymal wall analyses}

The pore pressure, pressure, von Mises stress and void ratio are important parameters for estimating the extent of damage in biological tissue. The pressure and void ratio at the centrum semiovale were approximately constant, despite the gradual increase in the TPG for both models, whereas the parameters increased significantly at the frontal and occipital horns. In fact, the two models did not show significant differences regarding the mechanical behavior of the tissue at the centrum semiovale; the main differences were found at the two horns, especially in terms of pore pressure (Table 3).

In SBG, the pore pressure decreased from 0 to $-1.0 \mathrm{mmHg}$ and $-0.8 \mathrm{mmHg}$ in the frontal and occipital horns, respectively, as the TPG increased from 0.1 to $1.0 \mathrm{mmHg}$. With the continued increase of TPG, the pore pressure at the frontal horn further decreased $(-1.1 \mathrm{mmHg})$, whereas it slightly increased at the occipital horn ($0.7 \mathrm{mmHg}$ ). However in $\mathrm{ABG}$, the pore pressure decreased from 0 to $-0.5 \mathrm{mmHg}$ and $-0.4 \mathrm{mmHg}$ in the frontal and occipital horns, respectively, as the TPG increased from 0.1 to $1.0 \mathrm{mmHg}$. With the continued increase of TPG, the pore pressure at the two horns slightly increased $(-0.3$ and $-0.04 \mathrm{mmHg}$, in the frontal and occipital horns, respectively). A negative pore pressure indicates fluid inflow, whereas zero or positive pore pressure indicates fluid free flow or fluid outflow. Thus, both models are capable of simulating fluid accumulation and a subsequent increase in volume, i.e., the periventricular lucency (PVL), as previously reported FE models have also demonstrated (Cheng and Bilston, 2010; Pena et al., 1999). However, in the SBG model, the continual increase of TPG to a maximum of $2.0 \mathrm{mmHg}$ resulted in a continued decrease in pore pressure, i.e., the continual steady inflow of fluid in the frontal horn. In contrast, the ABG model estimated an increase in pore pressure.

The increase in TPG was directly associated with ventricular enlargement. The results obtained with the SBG model suggested that the degree of PVL should be higher as the degree of ventricular enlargement becomes severe, at least for the frontal horn. In contrast, the results obtained with the ABG model suggest that as the degree of ventricular enlargement becomes greater over some certain level, the suctioning effect around the ventricular horns decreases. In reality, the prevalence of PVL is lower in severe ventricular enlargement 
(Asada et al., 1978). Although both the ABG and SBG models can simulate PVL, the results indicate that the ABG model is more suitable as a patient-specific FE brain model.

\subsection{The effects of the TPG increase on the cerebral cortex and subcortical structures}

Although the changes at the lateral ventricles and the ependymal wall deserve attention, additional findings were revealed at the cerebral cortex and subcortical structures. A deformation of the corpus callosum is often reported in NPH patients (Mataro et al., 2007). Damage to the corpus callosum can result in cognitive deterioration (Yamauchi et al., 1994) and gait disturbance (Jinkins, 1991), which are two of the three classic symptoms of NPH. Cognitive deterioration can be further aggravated if the damage caused by ventriculomegaly extends to the fornix (Hattori et al., 2012). The present study demonstrates that the degree of ventricular expansion is overestimated in the SBG model (Table 2). The SBG model also overestimated the stress on the corpus callosum and caudate nucleus (Figs. 5, 6). Although the role of the caudate nucleus in symptom development in NPH is unclear, it is well known that this structure is closely associated with various cognitive abilities (Mozley et al., 2001; Rogers et al., 2000; Volkow et al., 1998).

The characteristic upward elevation of the corpus callosum in NPH was not simulated in the ABG model or in the SBG model, as the present study utilized two-dimensional models. However, the two models did show deformations of the corpus callosum and the caudate nucleus (Fig. 5 and Table 3). Currently, deformation of these structures is thought to result from mechanical pressure due to ventriculomegaly (Mataro et al., 2007; Roricht et al., 1998). Although this may be true, a recent study found that the degree of deformation in the caudate nucleus and the corpus callosum is not correlated with the degree of ventriculomegaly (DeVito et al., 2007). Our models showed an increased pore pressure and a decreased void ratio, i.e., the outflow of fluid from the two structures. These results may indicate a thinning of the corpus callosum that may be partially caused by changes in the TPG.

The differences between the SBG and ABG models were most striking at the cortex. The behavior of the biomechanical parameters was similar to that in structures near the lateral ventricle (i.e., the caudate nucleus and the corpus callosum), and stress and pressure were overestimated in the SBG model. However, in the cortex, the SBG model underestimated the change in those two biomechanical parameters. The stress resulting from the increased TPG was almost linear in the SBG model, whereas the ABG model showed an exponential increase (Fig. 6C). It seems that brain models without sulci are not effective at TPGs over $1.5 \mathrm{mmHg}$, especially for simulating the degree of stress. The fact that the two models yield different results, especially in terms of the 
stress and pressure upon the cerebral cortex, may seem trivial because the two models have different sulcal geometrical characteristics. However, this difference also heavily affects the stress and pressure distribution on subcortical structures. The overall results clearly state the importance of including sulcal geometry in FE brain models for simulating a pathologic mass effect by space-occupying lesions.

\subsection{Limitations and suggestions}

The two-dimensional FE models presented here were sufficient for simulating the major radiological features of NPH and identifying differences in the mechanical changes between the anatomically detailed model and the model with simplified geometry. Nonetheless, this study has certain limitations that require clarification. The changes in the biomechanical properties in the two-dimensional model cannot appropriately reflect the pathological changes of a true hydrocephalic brain. Additionally, the geometrical details that the ABG model utilized were derived from a single healthy subject. In reality, sulcal variability among human brains is considerable, which may be better illustrated in a fully three-dimensional model. Finally, the brain anisotropy was neglected in this model; in reality, the brain tissue would react differently when subjected to changes in different directions. However, the model related to anatomical regions (i.e., white matter and gray matter) was defined by the homogenous mechanical properties of each mesh to avoid mathematical complications. Future studies may have to construct and employ a fully three-dimensional FE model incorporating the average anatomical geometry of human brains and dynamic nature of intracranial systems to reach a more realistic approximation.

\section{Conclusion}

Our findings demonstrate that the sulci play an important role in the biomechanical response of an FE brain model associated with space-occupying lesions (e.g., hydrocephalus). Conventional FE models, which lack geometrical details, significantly underestimate the pathologic changes that occur during the development of NPH. The integration of geometrical details provides a more accurate representation than conventional simplified FE models. This result may due to the geometrical characteristics of the sulci; the brain would be subjected to mechanical stress due to the growth of a space-occupying lesion. The cushioning effect the sulci provide is spatially distributed to compensate for the mechanical stress, thus avoiding immense local strain. Future FE studies for investigating pathologic changes in neurological disorders should consider using detailed models that balance anatomical accuracy and computational simplicity. 


\section{Acknowledgements}

This research was supported by the Basic Science Research Program through the National Research Foundation of Korea (NRF) funded by the Ministry of Science, ICT \& Future Planning (2013R1A1A1004827).

\section{Appendix}

The $C_{10}$ and $D_{1}$ parameters were needed to utilize the neo-Hookean hyper-elastic and volumetric porous material models. According to the Abaqus user manual (Systèmes, 2012), the strain energy potential $U$ for the compressible neo-Hookean material model is of the form

$$
\left.U=C_{10}\left(\overline{I_{1}}-3\right)+\frac{1}{D_{1}}(J-1)^{2},\left(\therefore J_{e l}=\frac{J}{J_{t h}}, J_{t h}=\left(1+e_{t h}\right)^{3}\right) \quad \text { Eq. (A. } 1\right)
$$

where, $J_{e l}$ is the elastic volume ratio, $J_{t h}$ is the thermal volume ratio, $e_{t h}$ is the linear thermal extension, $\bar{I}_{1}$ is the measure of the distortion in the material, and $C_{10}$ and $D_{1}$ are material parameters. The $C_{10}$ parameter describes the shear behavior of the material, and the $D_{1}$ parameter introduces compressibility. The tissue material is compressible, and thus $D_{1}$ should not be zero. The initial shear modulus, $\mu_{0}$, and the bulk modulus, $K_{0}$, are given by

$$
\mu_{0}=2 C_{10} \text { and } K_{0}=\frac{2}{D_{1}}
$$

Based on the above relationships, we can calculate $C_{10}$ and $D_{1}$ from typical material properties (i.e., Young's modulus and Poisson's ratio).

A porous material model can be expressed in the form of $\sigma=\mu f(\varepsilon)$ where the function of $\varepsilon$ is nonlinear and $\mu$ is a normalizing parameter. The porosity was obtained using Forchheimer's law and can be written as

$$
f=-\frac{k}{\gamma_{w}}\left(\frac{\partial u_{w}}{\partial x}-\rho_{w} g\right)
$$

where, $f$ is the volumetric flow rate, $k$ is the permeability, $\gamma_{w}$ is the wetting fluid specific weight, $u$ is the wetting fluid pore pressure, $x$ is the position, $\rho_{w}$ is the wetting fluid density and $\mathrm{g}$ is gravity. 


\section{References}

Adachi, M., Kawanami, T., Ohshima, F., Kato, T., 2006. Upper midbrain profile sign and cingulate sulcus sign: MRI findings on sagittal images in idiopathic normal-pressure hydrocephalus, Alzheimer's disease, and progressive supranuclear palsy. Radiat. Med. 24, 568-572.

Adams, R.D., Fisher, C.M., Hakim, S., Ojemann, R.G., Sweet, W.H., 1965. Symptomatic Occult Hydrocephalus with "Normal" Cerebrospinal-Fluid Pressure.A Treatable Syndrome. N. Engl. J. Med. 273, 117-126.

Asada, M., Tamaki, N., Kanazawa, Y., Matsumoto, S., Matsuo, M., Kimura, S., Fujii, S., Kaneda, Y., 1978. Computer analysis of periventricular lucency on the CT scan. Neuroradiol. 16, 207-211.

Battal, B., Kocaoglu, M., Bulakbasi, N., Husmen, G., Tuba Sanal, H., Tayfun, C., 2011. Cerebrospinal fluid flow imaging by using phase-contrast MR technique. Br. J. Radiol. 84, 758-765.

Biot, M.A., 1941. General Theory of Three-Dimensional Consolidation. J. Appl. Phys. 12, 155.

Bradshaw, D., Ivarsson, J., Morfey, C., Viano, D.C., 2001. Simulation of acute subdural hematoma and diffuse axonal injury in coronal head impact. J. Biomech. 34, 85-94.

Bulat, M., Lupret, V., Orehkovic, D., Klarica, M., 2008. Transventricular and transpial absorption of cerebrospinal fluid into cerebral microvessels. Coll. Antropol. 32 Suppl 1, 43-50.

Chan, K.H., Cheung, R.T., Fong, C.Y., Tsang, K.L., Mak, W., Ho, S.L., 2003. Clinical relevance of hydrocephalus as a presenting feature of tuberculous meningitis. QJM. 96, 643-648.

Cheng, S., Bilston, L.E., 2010. Computational model of the cerebral ventricles in hydrocephalus. J. Biomech. Eng. 132, 054501.

Clatz, O., Litrico, S., Delingette, H., Paquis, P., Ayache, N., 2007. Dynamic model of communicating hydrocephalus for surgery simulation. IEEE Trans. Biomed. Eng. 54, 755-758.

Cloots, R.J., Gervaise, H.M., van Dommelen, J.A., Geers, M.G., 2008. Biomechanics of traumatic brain injury: influences of the morphologic heterogeneities of the cerebral cortex. Ann. Biomed. Eng. 36, 1203-1215.

DeVito, E.E., Salmond, C.H., Owler, B.K., Sahakian, B.J., Pickard, J.D., 2007. Caudate structural abnormalities in idiopathic normal pressure hydrocephalus. Acta. Neurol. Scand. 116, 328-332.

Dusick, J.R., McArthur, D.L., Bergsneider, M., 2008. Success and complication rates of endoscopic third ventriculostomy for adult hydrocephalus: a series of 108 patients. Surg. Neurol. 69, 5-15.

Dutta-Roy, T., Wittek, A., Miller, K., 2008. Biomechanical modelling of normal pressure hydrocephalus. J. Biomech. 41, 2263-2271.

Faggin, R., Calderone, M., Denaro, L., Meneghini, L., d'Avella, D., 2011. Long-term operative failure of endoscopic third ventriculostomy in pediatric patients: the role of cine phase-contrast MR imaging. Neurosurg. Focus. 30, E1.

Feng, H., Huang, G., Liao, X., Fu, K., Tan, H., Pu, H., Cheng, Y., Liu, W., Zhao, D., 2004. Endoscopic third ventriculostomy in the management of obstructive hydrocephalus: an outcome analysis. J. Neurosurg. 100, 626-633.

Greitz, D., 1993. Cerebrospinal fluid circulation and associated intracranial dynamics. A radiologic investigation using MR imaging and radionuclide cisternography. Acta. Radiol. Suppl. 386, 1-23.

Greitz, D., 2004. Radiological assessment of hydrocephalus: new theories and implications for therapy. Neurosurg. Rev. 27, 145-165.

Greitz, D., Greitz, T., Hindmarsh, T., 1997. A new view on the CSF-circulation with the potential for pharmacological treatment of childhood hydrocephalus. Acta. Pædiatr. 86, 125-132.

Greitz, D., Hannerz, J., 1996. A proposed model of cerebrospinal fluid circulation: observations with radionuclide cisternography. AJNR. Am. J. Neuroradiol. 17, 431-438.

Hattori, T., Sato, R., Aoki, S., Yuasa, T., Mizusawa, H., 2012. Different patterns of fornix damage in idiopathic normal pressure hydrocephalus and Alzheimer disease. AJNR. Am. J. Neuroradiol. 33, 274-279.

Ho, J., Kleiven, S., 2009. Can sulci protect the brain from traumatic injury? J. Biomech. 42, 2074-2080.

Hoff, J., Barber, R., 1974. Transcerebral mantle pressure in normal pressure hydrocephalus. Arch. Neurol. 31, 101-105.

Iseki, C., Takahashi, Y., Wada, M., Kawanami, T., Adachi, M., Kato, T., 2014. Incidence of idiopathic normal pressure hydrocephalus (iNPH): A 10-year follow-up study of a rural community in Japan. J. Neurosurg. Sci. 339, 108-112.

Jinkins, J.R., 1991. Clinical manifestations of hydrocephalus caused by impingement of the corpus callosum on the falx: an MR study in 40 patients. AJNR. Am. J. Neuroradiol. 12, 331-340.

Jiroušek, O., Jíra, J., Jírová, J., Micka, M., 2005. Finite Element Model of Human Skull Used for Head Injury Criteria Assessment, In: Gilchrist, M.D. (Ed.), IUTAM Symposium on Impact Biomechanics: From Fundamental Insights to Applications. Springer Netherlands, pp. 459-467.

Kaczmarek, M., Subramaniam, R., Neff, S., 1997a. The hydromechanics of hydrocephalus: Steady-state solutions for cylindrical geometry. Bull. Math. Biol. 59, 295-323. 
Kaczmarek, M., Subramaniam, R.P., Neff, S.R., 1997b. The hydromechanics of hydrocephalus: steady-state solutions for cylindrical geometry. Bull. Math. Biol. 59, 295-323.

Kitagaki, H., Mori, E., Ishii, K., Yamaji, S., Hirono, N., Imamura, T., 1998. CSF spaces in idiopathic normal pressure hydrocephalus: morphology and volumetry. AJNR. Am. J. Neuroradiol. 19, 1277-1284.

Lefever, J.A., Garcia, J.J., Smith, J.H., 2013. A patient-specific, finite element model for noncommunicating hydrocephalus capable of large deformation. J. Biomech. 46, 1447-1453.

Malm, J., Lundkvist, B., Eklund, A., Koskinen, L.O., Kristensen, B., 2004. CSF outflow resistance as predictor of shunt function. A long-term study. Acta. Neurol. Scand. 110, 154-160.

Mataro, M., Matarin, M., Poca, M.A., Pueyo, R., Sahuquillo, J., Barrios, M., Junque, C., 2007. Functional and magnetic resonance imaging correlates of corpus callosum in normal pressure hydrocephalus before and after shunting. J. Neurol. Neurosurg. Psychiatry. 78, 395-398.

Miller, K., Taylor, Z., Nowinski, W.L., 2005. Towards computing brain deformations for diagnosis, prognosis and neurosurgical simulation. J. Mech. Med. Biol. 05, 105-121.

Mozley, L.H., Gur, R.C., Mozley, P.D., Gur, R.E., 2001. Striatal dopamine transporters and cognitive functioning in healthy men and women. Am. J. Psychiatry. 158, 1492-1499.

Mutoh, K., Okuno, T., Ito, M., Fujii, T., Mikawa, H., Moritake, K., Yamashita, J., Ishikawa, M., Kikuchi, H., 1988. Ipsilateral atrophy in children with hemispheric cerebral tumors: CT findings. J. Comput. Assist. Tomogr. 12, 740-743.

Nagashima, T., Tamaki, N., Matsumoto, S., Horwitz, B., Seguchi, Y., 1987. Biomechanics of hydrocephalus: a new theoretical model. Neurosurg. 21, 898-904.

Pena, A., Bolton, M.D., Whitehouse, H., Pickard, J.D., 1999. Effects of brain ventricular shape on periventricular biomechanics: a finite-element analysis. Neurosurg. 45, 107-116; discussion 116-108.

Poca, M.A., Mataro, M., Del Mar Matarin, M., Arikan, F., Junque, C., Sahuquillo, J., 2004. Is the placement of shunts in patients with idiopathic normal-pressure hydrocephalus worth the risk? Results of a study based on continuous monitoring of intracranial pressure. J. Neurosurg. 100, 855-866.

Pople, I.K., 2002. Hydrocephalus and shunts: What the neurologist should know. J. Neurol. Neurosurg. Psychiatry. 73, i17-i22.

Rogers, R.D., Andrews, T.C., Grasby, P.M., Brooks, D.J., Robbins, T.W., 2000. Contrasting cortical and subcortical activations produced by attentional-set shifting and reversal learning in humans. J. Cogn. Neurosci. 12, 142-162.

Roricht, S., Meyer, B.U., Woiciechowsky, C., Lehmann, R., 1998. Callosal and corticospinal tract function in patients with hydrocephalus: a morphometric and transcranial magnetic stimulation study. J. Neurol. 245, 280-288.

Stein, S.C., Guo, W., 2008. Have we made progress in preventing shunt failure? A critical analysis. J. Neurosurg. Pediatr. 1, 40-47.

Stephensen, H., Tisell, M., Wikkelso, C., 2002. There is no transmantle pressure gradient in communicating or noncommunicating hydrocephalus. Neurosurg. 50, 763-771; discussion 771-763.

Systèmes, D., 2012. Abaqus 6.12 Theory Manual. Dassault Systèmes Simulia Corp., Providence, Rhode Island.

Takhounts, E.G., et al, 2003. On the Development of the SIMon Finite Element Head Model. Stapp. Car. Crash. J. 47, 107-133.

Volkow, N.D., Gur, R.C., Wang, G.J., Fowler, J.S., Moberg, P.J., Ding, Y.S., Hitzemann, R., Smith, G., Logan, J., 1998. Association between decline in brain dopamine activity with age and cognitive and motor impairment in healthy individuals. Am. J. Psychiatry. 155, 344-349.

Wilkie, K.P., Drapaca, C.S., Sivaloganathan, S., 2010. A theoretical study of the effect of intraventricular pulsations on the pathogenesis of hydrocephalus. Appl. Math. Comput. 215, 3181-3191.

Wilkie, K.P., Drapaca, C.S., Sivaloganathan, S., 2012. Aging impact on brain biomechanics with applications to hydrocephalus. Math. Med. Biol. 29, 145-161.

Wirth, B., Sobey, I., 2006. An axisymmetric and fully 3D poroelastic model for the evolution of hydrocephalus. Math. Med. Biol. 23, 363-388.

Yamauchi, H., Fukuyama, H., Ogawa, M., Ouchi, Y., Kimura, J., 1994. Callosal atrophy in patients with lacunar infarction and extensive leukoaraiosis. An indicator of cognitive impairment. Stroke. 25, 1788-1793.

Yoganandan, N., Pintar, F.A., Larson, S.J., Sances, J.A., 1998. Frontiers in Head and Neck Trauma: Clinical and Biomechanical. IOS Press 


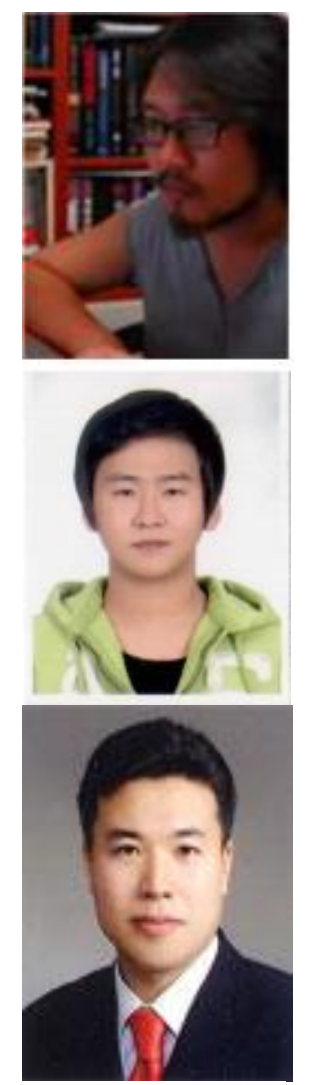

Hakseung Kim received his B.S. in brain and cognitive sciences (College of Information and Communication) and B.A. in Korean language and literature (College of Liberal Arts) from Korea University, South Korea (2013). He is currently pursuing his integrated M.S. \& Ph.D. degree in brain and cognitive engineering at the Department of Brain and Cognitive Engineering (Korea University). His specific areas of research include quantitative medical image analysis, patient monitoring in a critical care environment, cerebrospinal fluid dynamics, and patient-specific finite element modeling of neurological disorders associated with space-occupying lesion.

Dae-Hyeon Park received his B.E. in computer engineering from the Department of College of Engineering, Catholic University of Daegu (2012), South Korea. He is currently pursuing his integrated M.S. \& Ph.D. degree in brain and cognitive engineering at the Department of Brain and Cognitive Engineering (Korea University). His major research interest is finite element modeling of the human brain under mechanical stress from neurological disorders, such as hydrocephalus and idiopathic intracranial hypertension.

Seong Yi received his M.D. from the College of Medicine, Yonsei University (South Korea), and received his medical license in 1996. He began his special training in neurosurgery at Severance Hospital, Seoul, South Korea, in 1997 and obtained his M.S. and Ph.D. in neuroscience from Yonsei University (2008). He completed his residency (2001) and fellowship in spine surgery (2006) at the same institution, and later became an assistant professor in the Department of Neurosurgery, Yonsei University (2008). His professional interests include: spinal surgery, cervical artificial disc and development, minimally invasive spinal surgery, spinal cord tumor, spinal cord injury and transplantation of stem cells.

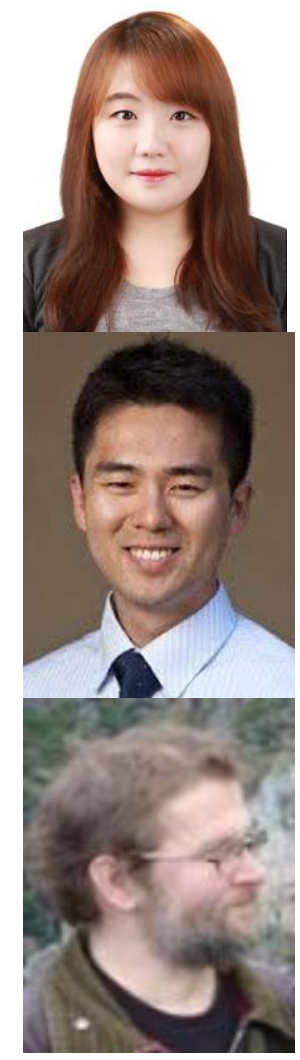

Eun-Jin Jeong received her B.E. in Biomedical Engineering (College of Health Science) and B.S. in brain and cognitive sciences (College of Information and Communication) from Korea University, South Korea (2015). She is pursuing her M.S. degree in brain and cognitive engineering in the Department of Brain and Cognitive Engineering at Korea University. Her research interests include: quantitative analysis of medical images obtained by various imaging modalities, finite element modeling of the brain with space-occupying lesions for identifying the pathogenesis of neurological disorders or predicting patient prognosis.

Byung C. Yoon grew up in South Korea and later moved to California. He completed his undergraduate studies at Johns Hopkins University and remained there to attend medical school. While in medical school, he decided to pursue a Ph.D. in Physiology, Development and Neuroscience at Cambridge University (Trinity College), UK, mentored by Christine Holt. He completed his Ph.D. thesis on localized protein translation in developing axons in 2010, and then earned his M.D. in Johns Hopkins School of Medicine, 2012. His major research interests are neuroregeneration and stem cells.

Marek Czosnyka is a Belvedere Professor of Technical Sciences, Ph.D. (Warsaw), D.Sc. (Warsaw) in Biomedical Engineering and a Professor in Brain Physics and Director of Neurosurgical Physics in the Neurosurgical Unit, University of Cambridge, UK. His specific areas of research include patterns of cerebrospinal fluid dynamics in hydrocephalus and pathophysiology of cerebrovascular dynamics in severe head trauma. His wide expertise and long list of publications in key neurosurgical journals have earned him great respect from neurosurgeons and physicists working in this field of research worldwide. 


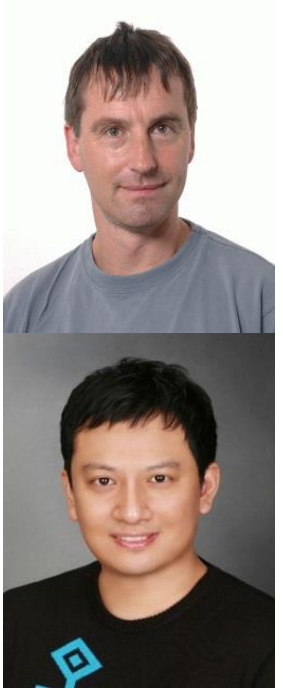

Michael Sutcliffe is a Reader in Mechanics of Materials in the Cambridge University Engineering Department, UK, and has been on the teaching staff there since 1992. He conducts various research projects on soft biological tissues in collaboration with medical colleagues at Cambridge's Addenbrooke's Hospital, as well as research with industrial partners into the engineering of composite materials and tribology.

Dong-Joo Kim received his Ph.D. in Engineering from the University of Cambridge, UK (2010), where his time was split between clinical research (in the Neurosurgery unit at Addenbrooke's Hospital) and brain modeling (in the Engineering Department). Afterwards, he spent two years as a research fellow in the Neuroscience Mental Health Program in the Department of Critical Care Medicine at the Hospital for Sick Children (University of Toronto), Canada. Since 2012, he has been an Assistant Professor in the Department of Brain and Cognitive Engineering at Korea University, South Korea. His areas of research include: brain mechanics, neuromonitoring and numerical neuro-modeling. 